\title{
Assessment of intestinal parasites among children taking antiparasitic drugs at Ngoma primary school, Rwanda.
}

\author{
Zéphanie Nzeyimana ${ }^{1}$, Thaddée Nshimiyimana ${ }^{2}$, Jean Marie Vianney Nyumbayire ${ }^{3}$, Filmin Uwihoreye ${ }^{4}$, \\ Osuwat Lawrence Obado ${ }^{1}$, Jean Baptiste Niyibizi, ${ }^{1,5,6^{*}}$ \\ ${ }^{1}$ Department of Medical Laboratory Sciences, Mount Kenya University Rwanda, Kigali campus \\ ${ }^{2}$ Department of Biomedical Laboratory Sciences, University of Rwanda, Rwanda \\ ${ }^{3}$ Laboratory Department, Masaka Hospital, Rwanda \\ ${ }^{4}$ Laboratory Department, Polyclinc Kanimba, Rwanda \\ ${ }^{5}$ Department of Biomedical Laboratory Sciences, Kibogora Polytechnic, Rwanda \\ ${ }^{6}$ Department of Biomedical Laboratory Sciences, Mount Kenya University, Kenya
}

\begin{abstract}
Background: Intestinal parasitic infections are amongst the most common infections worldwide. There is increasing recognition that these parasites can impair the growth and development of children of all ages. Since 2009, Rwanda MOH has targeted to reduce prevalence of soil transmitted helminths in school aged children from $65 \%$ to $50 \%$ by 2012 . Objectives: Our study aimed to assess intestinal parasites among Ngoma primary school children taking antiparasitic drugs (Albendazole and Mebendazole). The specific objectives of this study were to establish the most commonly encountered intestinal parasites in Ngoma primary school children and to determine intestinal parasite load among the infected children.

Methods: 120 stool samples, conveniently sampled, of Ngoma primary school children were examined for intestinal parasites. Slides were prepared directly for Wet mount in saline and iodine then microscopically examined. Finally, formalin-ether sedimentation techniques and Modified Wisconsin Sugar flotation method were used to concentrate stool samples for parasite identification and egg count respectively.

Results: Of the 120 children, 39 (32.5\%) were found infected with one or more intestinal parasites. The prevalence of Giardia lamblia, Entamoeba coli, Entamoeba histolytica, Ascaris lumbricoides, hookworm, and Taenia species infections as determined by Formol ether concentration technique were $13.3 \%, 7.5 \%, 5.8 \%, 3.3 \%, 1.7 \%$, and $.8 \%$ respectively. On the other hand, the mean of Egg per gram of stool estimates of Ascaris lumbricoides, hookworm, and Taenia species as determined by modified Wisconsin Sugar flotation method were 11, 9, and 6 egg per gram (epg), respectively. Conclusion: Intestinal parasitic infections were prevalent in varying magnitude among Ngoma primary school children. G. lamblia, E. coli, and E. histolyica together contribute to the majority of intestinal parasites encountered and they represent $86 \%$ of all the intestinal parasitic infections recovered.
\end{abstract}

Keywords: Parasites, Infestations, Cysts, Trophozoite, Diarrhea, Amoebiasis, Gastrointestinal infections.

Accepted February 20, 2018

\section{Introduction}

Intestinal parasites are organisms that live in the gastrointestinal tract of animals, including humans and can cause morbidity and mortality [1]. The two main types of intestinal parasites are helminths and protozoa. Helminths are worms with many cells. In their adult form, helminths cannot multiply in the human body. Protozoa have only one cell, and can multiply inside the human body, which can allow serious infections to develop [2]. The parasites in the intestines are classified into two classes such as protozoa and helminths. Protozoa are single celled and includes Amoebae, flagellates, ciliates, and coccidia and microsporodia whereas helminths multicellular organisms which include Nemathelminthes and Platyhelminthes [3]. Intestinal parasites are usually transmitted when someone comes in contact with infected feces for example, through contaminated soil, food, or water [4]. Certain types remain in the intestines; others travel outside the intestines to invade other organs. Most tapeworms and roundworms develop in the human body and lay their eggs there. The eggs then pass out of the body through feces and can infest others [5].

The World Health Organization (WHO) estimates that 3.5 billion people worldwide are infested with some type of intestinal parasite, and as many as 450 million of them are sick as a result. Children are most frequently infected with these parasites [1]. Intestinal parasitic infections are amongst the most common infections worldwide. It is estimated that 3.5 billion people are affected by intestinal parasites, and that 450 million are ill as a result of these infections, with the majority being children [1]. Neglected tropical diseases (NTDs) are the most common infections of poor people worldwide. In southern 
Rwanda in 2001, it was reported that $55 \%$ of 2004 school aged children were infected with hookworm, Ascaris lumbricoides, or Trichuris trichiura [6]. There is increasing recognition that these parasites can impair the growth and development of children of all ages [6]. These infections are regarded as a serious public health problem, as they cause iron deficiency anemia, growth retardation in children and other physical and mental health problems [5]. Intestinal parasites in primary schools are a major public health problem in Rwanda. It is believed that in Rwanda, more than $75 \%$ children were infected by intestinal worms in 2007 [7]. Highest prevalence of neglected diseases is found in tropical and subtropical regions [5]. In industrialized countries, risk groups include male homosexuals, travelers and recent immigrants, and institutionalized populations [5]. Tapeworms, pinworms, and roundworms are among the most common helminths in the United States [5].

From 2006, the Rwanda $\mathrm{MOH}$ has organised a biennial mother and child health week and in 2007, MOH began to administer Albendazole/Mebendazole, or Praziquantel (in bilharziosis risk zone) to school children aged between 5 and 16 years [4]. Since 2009, Rwanda MOH has targeted to reduce prevalence of soil transmitted helminths in school aged children from $65 \%$ to $50 \%$ by 2012 . This study aimed to assess intestinal parasitic infections among Ngoma primary school a children taking antiparasitic drugs (here is Albendazole). Despite efforts by the Rwanda $\mathrm{MOH}$ to reduce parasitic infections among primary school children, intestinal parasitic infections are still reported to be a major health problem among those children as evidenced by the more than $75 \%$ Rwandan children who were estimated to be infected by intestinal worms in 2007 [7]. More so, according to the Health Sector Strategic Plan of July 2009-June 2012, Rwanda MOH targeted to reduce prevalence of soil transmitted helminths in school aged children from $65 \%$ in 2009 to $50 \%$ by 2012 [8]. With this period of the strategic plan, evidently, the results were not as earlier anticipated. This study aimed to assess intestinal parasitic infections among Ngoma primary school children taking antiparasitic drugs (Albendazole/ Mebendazole) in Ngoma primary school located in Southern province, Huye district in order to decipher the most common intestinal parasites among Ngoma Primary School children taking antiparasitic drugs and also to determine the parasite load (parasite per gram of stool) among infected children of Ngoma primary school.

\section{Methods}

The study was carried out at Ngoma primary school which is located at the Southern province, Huye district, in Ngoma sector, $1.5 \mathrm{~km}$ from Huye town. Cross-sectional investigation was used to collect information concerning intestinal parasitic infections in Ngoma primary school. Ngoma Primary school (from $3^{\text {rd }}$ to $5^{\text {th }}$ year primary school students) children, aged between 8 and 14, taking antiparasitic drugs biennially administered were considered in this study. Eligible children were conveniently selected and samples from 120 children were tested for intestinal parasites. During sample collection and processing, each child was given a wide mouthed container with a wooden applicator stick and hygienic paper to collect required amount of fecal sample after explaining to them how and how much sample to be collected. Samples were labelled with child's code number corresponding to his/her data sheet then transported directly to Gatagara high school laboratory for analysis.

For macroscopic examination of specimens, specific features were taken into account in order to ascertain accuracy for each specimen's result. These include: the consistency of stool such as formed, soft, loose or watery; presence of blood and or mucus, presence of round worms, thread worms or tapeworm proglottids. After macroscopic examinations, the microscopic examination was carried out using two methods, which are wet mount preparation that were performed according to the Centres for Disease Control and Prevention (CDC) protocols (CDC, 2016). The second method was concentration technique in which the researchers used Formol-ether sedimentation technique to concentrate negative samples with wet mount method as described by CDC, 2016. Modified Wisconsin Sugar flotation method as described by Dr. Nolan in 2004 was used for egg count [9]. These results were recorded on the developed laboratory sheet. Data were analysed and presented in terms of percentages, tables, charts and figures generated using Microsoft excels. Results of mean average analysis, standard deviation were analyzed using SPSS version 20.

\section{Ethical Consideration}

This study was conducted after obtaining an informed consent from the school director and teachers of $3^{\text {rd }}$ to $5^{\text {th }}$ year primary school students of Ngoma Primary School, following the ethical approval given by Kigali Health Institute to carry out this research. The researchers clarified the purpose of the study and the participation of the children was voluntary. The identity of the children remained confidential, hence it was not exposed to the public.

\section{Results}

In Table 1 and Figure 1 respectively, represent age and sex distribution of the children enrolled and tested during the study. Less than half(Figure 2) of the tested children had soft stool from which a majority of parasites were recovered (Figure 3). Out of 120 examined children, 39 (32.5\%) had intestinal parasitism (Figures 4-6). Of the 39 children who had parasite infections, 6 species (Table 2) of parasites were identified, with Giardia

Table 1. Age distribution of the studied children

\begin{tabular}{|c|c|c|c|}
\hline Child age(years) & Frequency & Percent (\%) & Cumulative Percent (\%) \\
\hline $8-11$ & 73 & 60.8 & 60.8 \\
\hline $12-14$ & 47 & 39.2 & 100 \\
\hline Total & 120 & 100 & \\
\hline
\end{tabular}

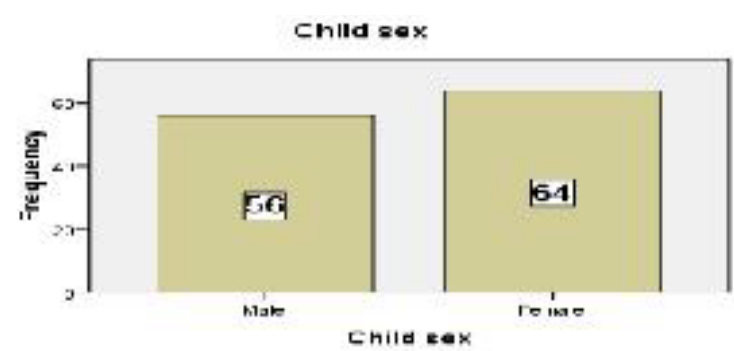

Figure 1. Distribution of Children by sex. 


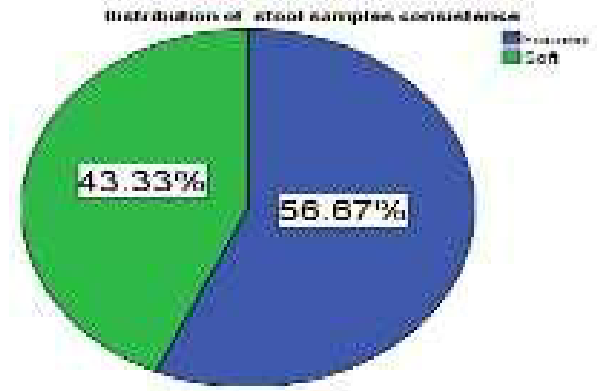

Figure 2. Distribution of stool specimen by their consistence studied stool were $56.67 \%$ formed and $43.33 \%$ soft.

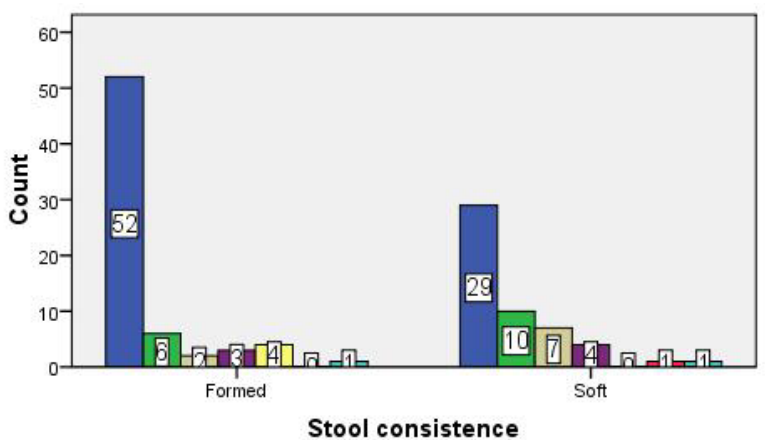

Identified parasites

No identified parasites

Giardia lamblia

Entamoeba coli

Entamoeba histolitica

Ascaris lumbricoides

Taenia species

Stool consistence

Figure 3. Distribution of identified parasites by stool consistence of studied children.

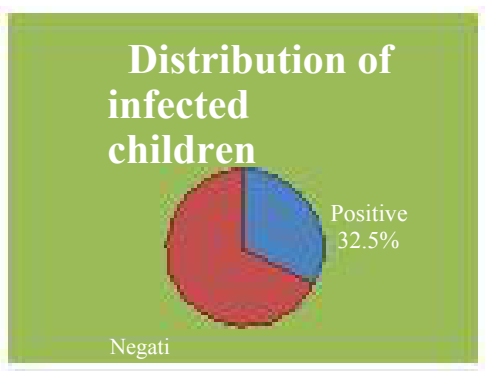

Figure 4. Distribution of infected children.

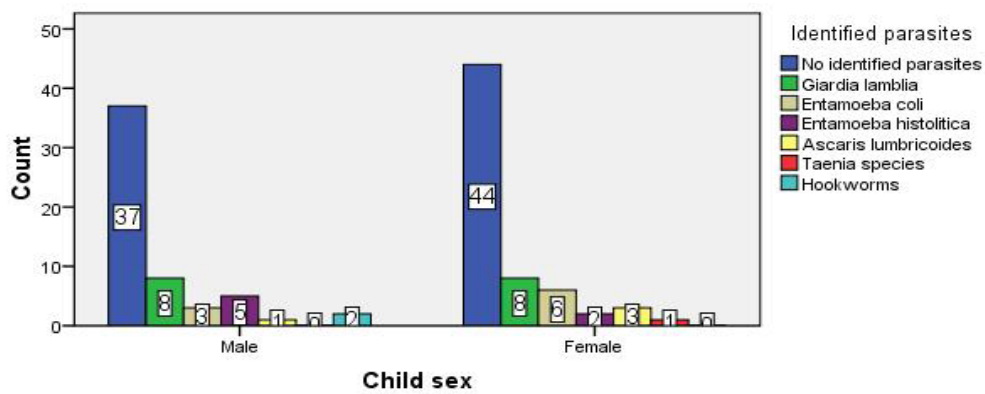

Figure 5. Distribution of identified parasites by sex of studied children.

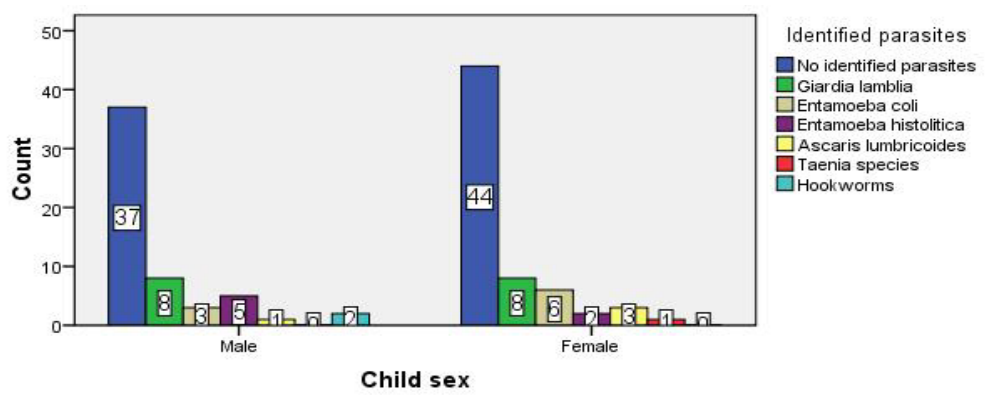

Figure 6. Distribution of identified parasites by child age of studied children. 
Citation: Nzeyimana Z, Nshimiyimana T, Nyumbayire JMV, et al. Assessment of intestinal parasites among children taking antiparasitic drugs at Ngoma primary school, Rwanda. J Parasit Dis Diagn Ther. 2018;3(1):1-5. DOI: 10.4066/2591-7846.1000020

lamblia being the most common (13.3\%) and Taenia spp the least $(.8 \%)$ encountered species. Of the 6 identified species, intestinal protozoa represent $86 \%$ whereas the remaining represents the intestinal helminths (Figure 7). Table 3 shows the parasite load/ egg per gram (epg) of the 3 parasite species from which eggs were recoverable.

Ascaris lumbricoides represented the highest epg (Mean of 11).

Our studied population aged between 8 and 14 years old. Studied ages were grouped into two groups, 8-11 and 12-14 representing respectively $60.8 \%$ (73) and $39.2 \%$ (39.2).

In 120 cases, 81 were not presenting any parasite and 39 presented any species of parasite identified being Giardia lamblia, Entamoeba coli, Entamoeba histolytica, Ascaris lumbricoides, Taenia species, or hookworms.3.5 Distribution of identified parasites by stool consistence of studied children.

Most identified parasitic stages were cystic and egg for protozoa and helminths respectively, as formed and soft feces are highly likely to contain these parasitic forms (CDC DPDx 2010). Soft stool presented more parasites than formed one.

In 64 counted females, 20 had any one of the intestinal parasites and 44 with no identified parasite whereas in 56 males, 19 had any one of the parasites and 37 with no identified parasite. Females presented more Entamoeba coli than males.

Giardia lamblia is the most prevalent intestinal parasite found in all studied ages. 12 years aged children present most children with no identified parasite.

- E. coli prevalent in 8 and 9 aged children.

- E. histolitica is mostly distributed in 10 and 11 aged children.

Table 2. Laboratory results of identified intestinal parasites in tested Ngoma primary school children.

\begin{tabular}{|c|c|c|c|}
\hline Laboratory findings & Frequency & Percent (\%) & $\begin{array}{c}\text { Cumulative } \\
\text { Percent }\end{array}$ \\
\hline No identified parasites & 81 & 67.5 & 67.5 \\
\hline Giardia lamblia & 16 & 13.3 & 80.8 \\
\hline Entamoeba coli & 9 & 7.5 & 88.3 \\
\hline Entamoeba histolitica & 7 & 5.8 & 94.2 \\
\hline Ascaris lumbricoides & 4 & 3.3 & 97.5 \\
\hline Taenia species & 1 & 0.8 & 98.3 \\
\hline Hookworms & 2 & 1.7 & 100 \\
\hline Total & $\mathbf{1 2 0}$ & $\mathbf{1 0 0}$ & \\
\hline
\end{tabular}

שiardia lamblia $\quad$ Antamoeba coli
Entamoeba histolytica $\square$ Ascaris lumbricoides
- Taenia species

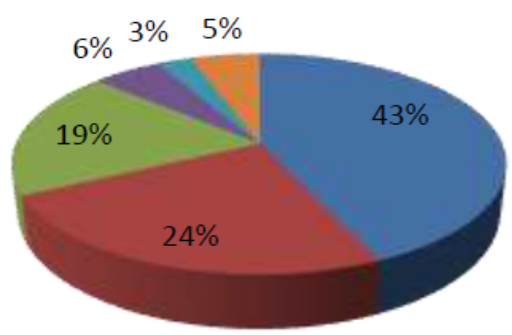

Figure 7. Prevalence of identified parasite species.
Table 3. Results for egg count.

\begin{tabular}{|c|c|c|c|}
\hline Identified helminths & Mean & N & Std. Deviation \\
\hline Ascaris lumbricoides & 11.25 & 4 & 2.217 \\
\hline Hookworms & 9.5 & 2 & 2.121 \\
\hline Taenia species & 6 & 1 &. \\
\hline Total & 10 & 7 & 2.646 \\
\hline
\end{tabular}

\section{Comparison of Parasite Species Proportions}

Giardia lamblia represents the highest proportion (43\%) followed by Entamoeba coli (24\%) and Entamoeba histolytica (19\%). Giardia lamblia, Entamoeba coli and Entamoeba histolytica are intestinal protozoa which are mostly encountered in Ngoma Primary school they all represent $86 \%$ of identified intestinal parasites whereas Ascaris lumbricoides, Hookworms, and Taenia species which are intestinal helminths represent only $14 \%$.

\section{Results for egg count}

The means of egg per gram of stool is as shown in Table 3 below.

The above table shows that 7 children were found to have helminthe eggs in their stool, among them, 4 had Ascaris lumbricoides eggs with mean of 11 egg per gram of stool (EPG), 2 with 9 hookworm EPG and 1 with 6 Taenia species EPG.

\section{Discussion}

In this study was conducted to establish the most commonly encountered intestinal parasites in Ngoma primary school children, and to determine the parasite load among helminths infected children. The results from this study revealed that $G$. lamblia is the most prevalent (13.3\%) intestinal parasitic infection among the children of Ngoma primary school. Along with $E$. coli (7.5\%) and E. histolyica (5.8\%), G. lamblia predominantly infects children aged 8-11 years, with much less infection seen among the older (12-14 years) children. These findings are comparable to those reported from an Indian study by Wani et al. The Indian study was conducted among school children of Srinager city. Wani et al., reported Ascaris lumbricoides as the most prevalent (28.4\%) intestinal parasite with Giardia lamblia a distant third at $4.9 \%$. Comparably a study in Kassala town (Sudan) reported a Giardia lamblia prevalence of $12.3 \%$ and Entamoeba histolytica $.4 \%$. In a Rwanda study that included 2400 children aged 2-20 years old with $93 \%$ being 5-10 years old, the overall intestinal prevalence was $55.9 \%$. These findings were remarkably different from ours in that, hookworms constituted $44.5 \%$ of the parasites. As was variously explained hygiene is at the heart of all these glaring and unacceptably high prevalence rate. Whereas this study report mainly high parasite load (epg) of Ascaris lumbricoides (11 epg), Hookworms (9 epg), and Taenia species (6 epg) several other studies have reported high parasitic loads. The authors suggest that the major difference arises from sample size (120) which was estimated to be low compared to the one conducted by Kassala with 900 children as sample size and the Rwanda study by Kabatereine with a sample size of 2400 children. Whereas females appeared to have higher infection rates than male students, the present authors could not attribute this occurrence to any particular or specific reason. Increase Ascaris lumbricoides and Hookworm 
species parasite loads have also been equally reported among those age groups. Cyst and eggs were mainly recovered from children who had formed stool as expected (CDC DPDx 2010).

The present study reports that intestinal protozoa represent the highest prevalence $(26.6 \%)$ in identified intestinal parasites. This can be attributed to the type of deworming drug (albendazole) administered which targets only intestinal helminths which have significantly decreased to $5.8 \%$ in the studied children as $\mathrm{MOH}$ targeted to reduce intestinal helminths from $65 \%$ to $50 \%$ [8-16]. This can be probably attributed to increased awareness to prevent intestinal parasites, studied low sample size and the town location of Ngoma Primary school. In the present study, authors also report that mean epg, burden of the cases is positively related with the infection rate, of 11 (below 200) is of light infections as WHO report of 2002. WHO reported that less than 200 epg represent light infections for hookworms whereas greater than 200 epg should be regarded as a potential danger. This can be attributed to the Rwanda $\mathrm{MOH}$ deworming program, and asymptomatic tested children.

\section{Conclusion}

Intestinal parasitic infections are still a major health problem among the children we studied.

G. lamblia, E. coli and E. histolyica together contribute to the majority of intestinal parasites encountered and they represent $86 \%$ of all the intestinal parasitic infections recovered. However, our findings seem to suggest that the $\mathrm{MOH}$ interventional strategies are playing a positive role as there seems to be a decline in prevalence of intestinal helminths. Because of what appears to be a decline in intestinal parasite prevalence, $\mathrm{MOH}$ should continue with the deworming exercise. However antiprotozoal drugs should be integrated for protozoa are prevailing. Laboratories need to be equipped with better stool concentration; both flotation and sedimentation techniques and personnel trained on how to use them. Hygiene practices need to be emphasized, particularly to the younger children. We would also recommend further studies using larger sample sizes and better diagnostic approaches.

\section{Limitations of the Study}

In the present research, due to lack of Zinc sulphate reagents, Zinc sulphate floatation technique was not carried out.

\section{Conflict of Interest}

The authors declare that there is no conflict of interest regarding the publication of this paper as far as this work is concerned.

\section{Acknowledgement}

Authors kindly appreciate the contribution of University of Rwanda, former KHI administration, Osuwat Lawrence Obado, Rwanda Education Board and Ministry of education at large.

\section{References}

1. Lynne SG. Classification of Human Parasites, Vectors, and Similar Organisms. Clinical Infectious Diseases. 1993;16:614-15.
2. Curtis V, Cairncross S, Yonli R, et al. Review: Domestic hygiene and diarrhoea-pinpointing the problem. Tropical Medicine and International Health 2000;5:22-32.

3. Madigan MT, Martinko JM, Parker J. Brock Biology of Microorganisms. Pearson Education Inc. 2006;947-948.

4. Elmer WK. Colour Atlas and Textbook of Diagnostic Microbiolog. Lippencort 1997;1071-1162.

5. Dawit A. Medical parasitology: Lecture notes Degree and Diploma Programs For Health Science Students 2004.

6. Eleonor TB, Vicente YB, Winifreda U. Infection status of intestinal parasites in children living in residential institutions in Metro Manila, the Philippines. Korean J Parasitol. 2004;41:113-5.

7. Beaver PC, Jung RC, Cupp EW. Clinical Parasitology. Lea and Febiger. 1984.

8. Ministry of Health: Rwanda ministry of Health: Health Sector Strategic Plan July 2009-June 2012.

9. Formalin-Ethyl Acetate Sedimentation and Zinc Sulfate Flotation Techniques for Detection of Intestinal Parasites. J Clinical Microbio. 1981;13:882-4

10. https://www.cdc.gov/dpdx/diagnosticprocedures/stool/ microexam.html

11. Haddad CM. Imaging of parasitic disease. J Media Reviews. 299:2691-2.

12. Kim BJ, Ock MS, Chung DI, et al. The intestinal parasite infection status of inhabitants in the Roxas city, the Philippines. Korean J Parasitol. 2003;41:113-5.

13. Amal KM, Anthony RM. Neglected Tropical Diseases: Epidemiology and Global Burden. Tropical Medicine and Infectious Disease. 2017; 2:36; doi:10.3390/ tropicalmed2030036

14. http://www.theaccessproject.com/index.php/about/ntd/

15. Ichhpujani RL, Bhatia R. Medical Parasitology. $2^{\text {nd }}$ Ed Jaypee Bros, New Delhi, 1998.

16. Abdelsafi AG, Mohammed AE. Prevalence of Intestinal Parasite Infection in Primary School Children in Elengaz Area, Khartoum, Sudan. Academic Research International. 2014; 5: 2223-53.

\section{*Correspondence to:}

Jean Baptiste Niyibizi

Department of Medical Laboratory Sciences,

Mount Kenya University Rwanda,

Kigali campus

E-mail: niyibizi3@gmail.com 